\title{
Study of the Non-Immune Hydrops Fetalis (NIHF) and its Management
}

\author{
Ramani TV1* and Saritha $\mathrm{S}^{2}$ \\ ${ }^{1}$ Assistant professor, Kamineni Academy of Medical Sciences \& Research Center, India \\ ${ }^{2}$ Professor \& HOD, Kamineni Academy of Medical Sciences \& Research Center, India \\ *Corresponding author: Ramani TV, Kamineni Academy of Medical Sciences \&
}

\section{Research Article}

Volume 2 Issue 1

Received Date: March 24, 2018

Published Date: April 16, 2018

Research Center, LB Nagar, Hyderabad, India, Email: ramani_muddaloor@hotmail.co.uk

\section{Abstract}

Hydrops Fetalis is a Greek term that describes pathological fluid accumulation in fetal soft tissues and serous cavities, peritoneal, pleural, pericardial, scalp and body wall. Hydrops Fetalis is considered as a prenatal form of cardiac failure. It is broadly divided into two groups -Immune Hydrops Fetalis (IHF) and Non -Immune Hydrops Fetalis (NIHF). In previous years most of the Immune Hydrops Fetalis is caused by Erythroblastosis Fetalis secondary to Rh Iso-immunization with presence of maternal circulating red cell antibodies. IHF has now decreased with passive maternal immunization markedly up to $20 \%$ (1990).

Recently, Non- immune Hydrops Fetalis (NIHF) is relatively more common and accounts for 90\% of Hydrops Fetalis. NIHF is also defined as universal edema unassociated with Erythroblastosis Fetalis. It can be caused by wide variety of factors including fetal disorders (Chromosomal abnormalities, Congenital malformation \& Twinning), Cardiovascular, metabolic causes, maternal diseases (Diabetes, Hypothyroidism, Anemias), placental and cord abnormalities and infectious pathogens acquired in-utero (Cytomegalovirus). The incidence of NIHF is estimated at 1 in 3000 pregnancies. The aetiology of Hydrops Foetalis is based on the presence or absence of anaemia leading to heart failure and generalized oedema in the foetus and neonate. In approximately $1 / 4$ of all cases, the cause is not determined.

Keywords: Non-immune Hydrops Fetalis (NIHF); Immune Hydrops Fetalis (IHF); Edema; Pericardial effusion; Peritoneal effusion and Erythroblastosis Fetalis

\section{Introduction}

Hydrops Fetalis is an accumulation of extracellular fluid in fetal body civilities like pleural, pericardial, scalp and in body wall [1]. Ballantyne described the first case of
Hydrops Fetalis over 100 years ago, later NIHF was first distinguished from IHF by Edit Potter in 1943. He described NIHF as universal edema other than $\mathrm{Rh}$ incompatibility [2]. NIHF is associated with accumulation of extracellular fluid in fetal body cavities like pleural, 
pericardial, scalp, body wall with increased skin thickness of more than $5 \mathrm{~mm}$ due to generalized subcutaneous edema and placental enlargement. When first described HF constituted $20 \%$ of all cases, but with effective anti D prophylaxis for immune Hydrops, NIHF constitutes $90 \%$ cases of fetal Hydrops [3]. NIHF incidence reported as 1 in 3000 pregnancies [4]. Approximately $50 \%$ of fetuses with non-immune hydrops fetalis die in utero, and about half of the live born infants survive.

Routine ultrasound has been recommended as the initial diagnosis of NIHF. Placental thickness should be measured in all cases to rule out placental edema. 3D scan helps in determination of Facial dysmorphology which may give clue to inborn errors of metabolism and Cystic hygroma may be in cases of Aneuploidy, fetal echocardiography, examination of maternal blood for fetal erythrocytes, amniocentesis and sampling of fetal blood. We report two of NIHF fetuses about 16-18 weeks of gestational age.

\section{Materials and Methods}

The present study was conducted over a period of one year among 1000 live births in Kamineni academy of medical sciences and research Centre. We found two cases of Non- immune Hydrops Fetalis (NIHF) of 16-18 weeks. Hydrops Fetuses are discovered incidentally during Prenatal Ultra sonography in the first and second trimester. The incidence, etiology, management options regarding NIHF were discussed in this article.

\section{Observations and Results}

Fetus- 1: was a Male NIHF about 16-18 weeks (Figure $1 \& 2)$.

\section{Obstetric history}

A 28 years old mother G3P2L2 with 16-18 weeks of gestation came to the hospital and diagnosed IUD. The First pregnancy was full term, normal vaginal delivery, female 6yrs and is healthy. The second pregnancy was full term, normal vaginal delivery, female 3yrs healthy. The present case was Third pregnancy, spontaneous conception with 18 weeks of gestation. The BP was $110 / 70 \mathrm{mmHg}$, pulse rate $82 / \mathrm{min}$, blood group was A +ve and $\mathrm{Hb}-5.3$ gms. The mother with severe anemia and gave history of viral infection during early pregnancy.

P/A Examination: Uterus 20 weeks and FHS was absent.

\section{USG Report}

a. Single fetus with no cardiac activity and no fetal movements. b. Cystic hygromatous changes around the neck.

c. Signs of Fetal Hydrops changes: Oedma is seen in the fetal subcutaneous tissue and with Scalp edema.

d. Fetus $-16+$ weeks, weight 144 gms.

e. Over riding of small bones of skull.

f. Liquor adequate. Placentomegaly, Fundal, Posterior, and with Grade 1 maturity.

Impression: Intra uterine fetal demise with gestational age of 16 weeks 2days, with Hydrops changes. After admission consent was taken for MTP. She spontaneously expelled dead fetus weight 180 gms with edematous and hydrops changes. Placenta and membranes expelled and weighing $140 \mathrm{gms}$. The dead fetus was send to the Anatomy department KAMS\& RC for academic purpose.

Impression: Male fetus with Non-immune Hydrops fetalis and cystic hygromatous changes around the neck with Facial dysmorphology (Figure 1\&2).
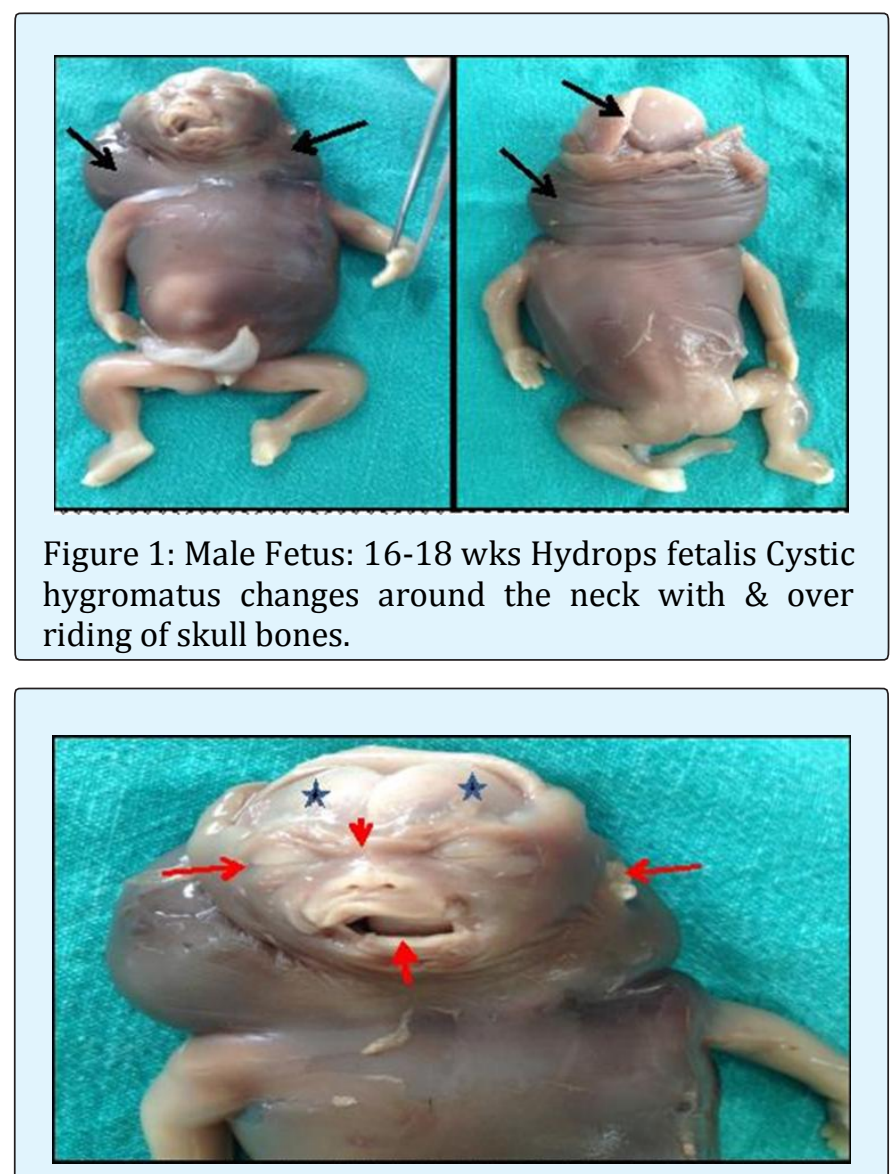

Figure 2: Facial dysmorphology: Hypertelorism, low set ears, flat nasal bridge \& macrognathia. Skin over scalp withdrawn\& parietal bones are visualized. 
Fetus 2: was a Female NIHF about 16-18 weeks, with multiple congenital anomalies and mother underwent MTP (Figures 3 \& 4).

Obstetric history: A 22year old mother G3P1L1 with 1617 weeks of gestation. The First pregnancy was full term, normal vaginal delivery, and male baby of 18 months old and healthy. The Second pregnancy was a twin gestation of 12 weeks induced abortion for fetal anomalies. The present pregnancy was a spontaneous conception with 16-18 weeks of gestation. The BP was $120 / 80 \mathrm{mmHg}$, pulse rate $82 / \mathrm{min}$, blood group was $\mathrm{A}+\mathrm{ve}$ and $\mathrm{Hb}-7.2$ gms.

P/A Examination: Uterus 16-18 weeks and FHS +ve.

\section{Tiffa Scan Report}

a) Single viable fetus with fetal movements. Generalized subcutaneous edema of the entire body with presence of bilateral pleural and pericardial effusion. Signs of Fetal Hydrops changes.

b) Brain: Choroid plexus cyst noted in left lateral ventricle. Multi-loculated cystic lesion noted in the neck not communicating with cranium suggestive of cystic hygroma.

c) Thorax: Both lungs appear echogenic with pleural effusion. Heart: Complex congenital anomaly with single ventricle and mild pericardial effusion. Heart rate: $167 \mathrm{BPM}$.

d) Fetus 17+ weeks, weight 147gms.

e) Liquor adequate. Placenta Fundal, Anterior, Grade 1 maturity.

Diagnosis: Single live Intra uterine fetus with gestational age 16 weeks 5 days, with Hydrops fetalis and multiple congenital anomalies with facial dysmorphology.

After admission consent was taken for MTP. She underwent medical abortion and expelled fetus and placenta. The Fetus was edematous with Hydrops changes. Placenta and membranes expelled and was 160 gms. The dead fetus was send to Anatomy department KAMS\& RC for academic purpose.

Impression: Female fetus with Non- immune Hydrops fetalis and cystic hygromatous changes around the neck.

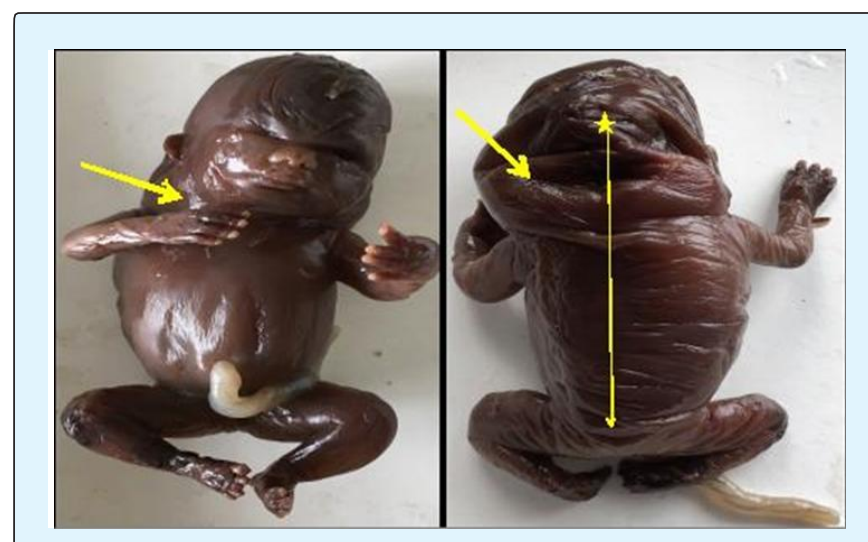

Figure 3: Female Fetus 2-16-18 Weeks: Macerated Hydrops fetalis with cystic hygromarous changes around the neck \& skin on the back is wrinkled.

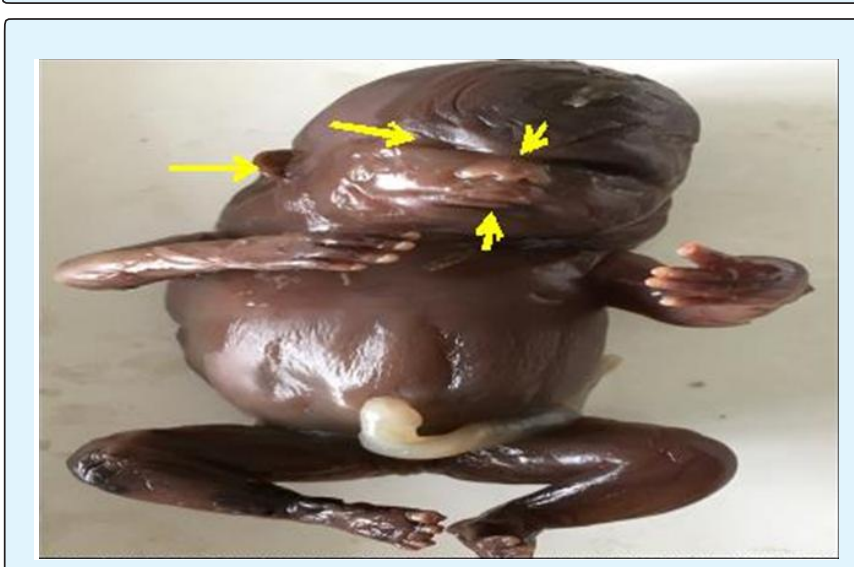

Figure 4: Facial dysmorphology Hypertelorism, flat nasal bridge, low set ears \& micrognathia.

\section{Discussion}

Hydrops fetalis is a Greek term, that describes pathological fluid accumulation in fetal soft tissues and serous cavities. The more common areas include fluid in the abdomen (ascites), fluid around the lungs (pleural effusion), fluid around the heart (pericardial effusion), or extra fluid under the skin edema (anasarca) or scalp. It is detected by foetal ultrasound if the presence of abnormal fluid collection and skin edema (skin thickness) $>5 \mathrm{~mm}$ [5]. Sonographic findings include Placental thickness $>4 \mathrm{cms}$ in second trimester or $>6 \mathrm{cms}$ in third trimester $[6,7]$. 
Hydrops fetalis are of two types. In previous years most of cases of Hydrops were caused by severe Erythroblastosis fetalis secondary to $\mathrm{Rh}$ isoimmunization. With passive maternal immunization, this type of Immune Hydrops fetalis (IHF) has decreased markedly from $100 \%$ to less than 20\%. According to Wilson, et al. [8] the commonest cause of Hydrops (IHF) was Rh isoimmunization and mortality rates were as high as $100 \%$ and fell to less than $20 \%$ by 1990 . Other studies shows that there are other materno-fetal blood group incompatibilities can still occur. The changing etiology presently most of the cases of Hydrops fetalis are now caused by Non immune Hydrops Foetalis (NIHF). Later Edith Potter in 1943 described NIHF with universal edema unassociated with Erythroblastosis which now accounts for $90 \%$ of cases of Hydrops fetalis [2,3]. The diversity of potential etiologies in the investigations of NIHF requires multi disciplinary approach. NIHF has a favourable outcome in $27.5 \%$ of cases with idiopathic NIHF carrying the worst prognosis. Negative prognostic factors described are enlarged biventricular dimension, early manifestation of pericardial effusion, and pulmonary hypoplasia secondary to pleural effusions. Pleural fluid drainage in utero may improve outcome in NIHF and timely performed thoracoamniotic shunting procedures are also associated with a $60 \%$ survival rate.

If Hydrops develops after 30 weeks of gestation, the mortality rate is approximately $25 \%$. If Hydrops develops before 30 weeks gestation, the mortality rate is more than $90 \%$.

\section{Pathogenesis}

The common Pathophysiology underlying the etiology of Hydrops fetalis of Nonimmune type is imbalance in regulation of fluid movement between the vascular and interstitial spaces. It may be increase in interstitial fluid production or decrease in lymphatic return [9]. The most common etiology of NIHF includes cardiovascular, chromosomal /genetic and hematologic abnormalities in the fetus. Others may be structural fetal anomalies of thorax, twinning complications, congenital hepatic cirrhosis, and congenital nephritic syndrome. All leading to severe cardiac failure in fetus. Approximately $50 \%$ of fetuses with NIHF die in utero with severe viral infections causing fetal anemia (Parvo virus B19or TORCH). Others include placental and cord abnormalities and inherited metabolic disorders which may also be cause NIHF [10]. New causes of NIHF are being reported every year but approximately in $50 \%$ of cases, no cause can be found despite extensive investigations [11]. Ultrasonography before 20 weeks gestation shows the first sign of increased nuchal translucency, followed by generalized skin edema, particularly at back, wherein skin thickness may be 15 to $20 \mathrm{~mm}$. Mostly associated with complex cardiac defects or abnormal karyotype like trisomy 18 or 21 [12]. Before 20 week of gestation, placental oedema is a consistent finding with skin edema and thickness over $25 \mathrm{~mm}$ is diagnosed as NIHF. Ultrasound measurement of placental thickness is considered for early diagnosis of alpha thalassemia. Maternal serum sample should be considered in cases of consanguineous marriage, stillborn and recurrent Hydrops [13]. An Inborn error of metabolism (IBM) causes Hydrops either by severe anemia or liver failure. This may account for $10 \%$ of total cases. Prognosis for IBM is poor leading to recurrent Hydrops being up to 25\%in subsequent pregnancy Facial dysmorphology may give clue to inborn errors of metabolism. Cystic hygroma may be related to an Aneuploidy (Trisomy 13,18 \& 21) or Turner syndrome [14-16].

Women with NIHF may develop Mirror syndrome, an uncommon complication in which mother develops edema that "mirrors" that of her Hydrops fetus. Mirror syndrome may represent a form of preeclampsia with edema, hypertension and proteinuria [17].

The current study reports two fetuses with NIHF associated with cystic hygroma and facial ysmorphology. The First fetus was a Male, about 16-18 weeks of gestation, with intrauterine death. A sonographic investigation identified the fetus as NIHF with cystic hygroma. The mother presented with severe anaemia and $\mathrm{h} / \mathrm{o}$ viral infection during pregnancy. The final impression was male fetus with $16-18$ weeks of gestation with NIHF and without any congenital anomalies. Main cause of NIHF probably may be severe anaemia and viral infection of the mother which may be spread to the fetus or no cause can be found despite extensive investigations. However Foetus had Cystic hygroma which may be suggestive of Aneuploidy (Trisomy 13,18 \& 21) or Turner's syndrome and Facial dysmorphology which is suggestive of an Inborn metabolic errors.

The Second FETUS was a female, about 16-18weeks of gestation identified NIHF with severe Cardiac abnormalities, CNS defects and cystic hygroma on ultra sound. Medical termination of pregnancy was performed. The final impression was female fetus with 16-18 weeks of gestation with NIHF. However this Foetus also had Cystic hygroma and Facial dysmorphology which is a clue for any Aneuploidy (Trisomy 13,18\&21) or Turner's syndrome or In born metabolic errors leading to multiple congenital anomalies. In both the cases the parents were advised for chromosomal and genetic analysis. 


\section{Conclusion}

NIHF requires multi disciplinary approach. Evaluation of Hydrops is by antibody screening (Indirect Coombs test) followed by detailed sonography. Ultra Sonographic identification of Hydrops fetalis and placenta can be determined in about $65-85 \%$ of cases although this includes post natal evaluation. Fetal chromosomal analysis and genetic molecular testing can be done to rule out any aneuploidy. Fetus with heart failure needs a multi vessel doppler study using umbilical artery or vein, middle cerebral artery or ductus venosus. Management is guided by presence or absence of additional anomalies. The prognosis depends on etiology and gestational age.

\section{References}

1. Ulrich S, Gruslin A (2003) Fetal Hydrops and Ascites. In: Diagnostic Imaging of fetal Anomalies. Nyberg(Ed), Pildelphia, pp: 713-743.

2. Potter EL (1943) Universal edema of fetus unassociated with erythroblastosis. Am J Obstet Gynecol 46(1): 130-134.

3. Santolaya J, Alley D, Jaffe R, Warsof SL (1992) Antenatal classification of Hydrops fetalis. Obstet Gynecol 79(2): 256-259.

4. Heinonen S, Ryynanen M, Kirkinen P (2000) Etiology and outcome of second trimester non immunologic fetal Hydrops. Acta Obstet Gynecol Scand 79(1): 1518.

5. Skoll MA, Sharland GK, Allan LD (1991) Is the ultrasound definition of fluid collections in non immune hydrops fetalis helpful in defining the underlying cause or predicting outcome? Ultrasound Obstet Gynecol 1(5): 309-312.

6. Lee AJ, Bethune $M$, Hiscock RJ (2012) Placental thickness in the second trimester: A pilot study to determine the normal range. J Ultrasound Med 31(2): 213-218.
7. Hoddick WK, Mahony BS, Callen PW, Filly RA (1985) Placental thickness. Ultra sound Med 4(9): 479-482.

8. Wilson DC, Halliday HL, Mc Clure G, Reid M (1990) The changing pattern of Hydrops fetalis. Ulster Med J 59(2): 119-121.

9. Bellini C, Hennekam RC (2012) Non immune Hydrops fetalis: a short review of etiology and pathology. Am J Med Genet A 158A(3): 597-605.

10. Apkon M (1995) Pathophysiology of Hydrops fetalis. Semin perinatol 19(6): 437-446.

11. Trainor B, Tubman R (2006) The Emerging pattern of Hydrops Fetalis - Incidence, etiology and management. Ulster Med J 75(3): 185-186.

12. Jauniaux E (1997) Diagnosis and management of early non immune Hydrops fetalis. Prenat Diagn 17(13): 1261-1268.

13. Sreelakshmi K, Roopa PS, Muralidhar VP Dept of ObGyn, Kasturba Medical College, Manipal 576104.

14. Jones DC (1995) Nonimmune fetal Hydrops: diagnosis and obstetrical management. Semin Perinatol 19(6): 447-461.

15. Huang HR, Tsay PK, Chiang MC (2007) Prognostic factors and clinical features in live born neonates with Hydrops fetalis. Am J Perinatol 24(1): 33-38.

16. Santo S, Mansour S, Thilaganathan B, Homfray T, Papageorghiou A, et al. (2011) Prenatal diagnosis of non-immune Hydrops fetalis: What do well the parents? Prenatal Diagn 31(2): 186-195.

17. Braun T, Brauer M, Fuchs I, Czernik C, Dudenhausen JW, et al. (2010) Mirror syndrome: a systematic review of fetal associated conditions, maternal presentation, and perinatal outcome. Fetal Diagn Ther 27(4): 191-203. 